\title{
Emergency Pharmaceutical Care in ED and ICU: Toxicology, Infectious Disease, Life Saving Drugs Management, Pharmacoeconomy as Synergic Knowledge Instrument to Reduce Mortality Rate and Healthcare Cost 2019
}

\author{
Mauro luisetto $^{1 *}$, Ghulam Rasool Mashori ${ }^{2}$ and Behzad Nili Ahmadabadi ${ }^{3}$ \\ ${ }^{1}$ Applied pharmacologist, European specialist in laboratory medicine Pc area, Italy \\ ${ }^{2}$ Department of Pharmacology, Pakistan
}

${ }^{3}$ Innovative Pharmaceutical product development specialist, USA

*Corresponding author: Mauro luisetto, applied pharmacologist, European specialist in laboratory medicine Pc area, Italy

\begin{abstract}
In this work we discuss about healthcare advantages related clinical pharmacist to take part in stabile way in medical team to improve clinical - economical outcomes (and the role played by clinical pharmacist with medicine laboratory and imaging tools to monitoring therapy). The role played by hospital clinical pharmacist in emergency department or in ICU or in other relevant wards like infectious disease and other is a real fact with reduction in mortality rate and right cost containment as reported in biomedical literature. The high pharmacist specialization in field like toxicology, antidothes, antimicrobial agents, nuclear medicine, imaging and laboratory medicine contribute in every medical team to better Obtain efficacy clinical outcomes.
\end{abstract}

\section{Introduction}

Ward Clinical pharmacy, pharmaceutical care, and consultant pharmacist service can be considered interesting instruments in the world of cognitive service to improve global clinical/ economical results in multi-disciplinary medical team. This pharmacist field of working can be considered as an opportunity in this time in with a great number of new drugs molecules, medical - diagnostic procedure added to the more complexity of cure, politherapy and more necessity to containment cost for drugs and medical devices than past. Reduction of medication therapy errors is needed and actively requested by patients and health organization institution and government. Multi-professional healthcare team is the right keywords in today health- care systems. Clinical pharmacist contributes today in many disciplines like oncology, toxicology, emergencies medicine, ICU, nuclear medicine, infectious disease, nephrology, nutrition service and other. To be developed

pharmacist competence in field of Emergency medicine, ICU, toxicology, imaging, medicine laboratory , biochemistry molecular biology, genetic, immune-histochemistry for relationship in pharmacological therapy and in new biological agents. Management of new innovative pharmacological- therapies and the necessity of cost containment represent a key to make the difference in medical TEAM. At the same time clinical service as consultant out of hospital settings is a great opportunity for patients and their needs. A consultant pharmacist for patient in the complex world of drugs therapies can be referent point in the different medical speciality and in the complex health care world. The specific pharmaceutical chemist and pharmacology competence conjugated with clinical activity can give an improving in clinical some outcomes, in patient health and quality of life. A reduction in mortality rate is an undeniable fact. Also, economic monitoring of therapies costs 
receive benefit in this approach. Emergency department and ICT are setting with high complexity of patient and the clinical pharmacist can add specific professionality to improve also clinical outcomes.

\section{Material and Methods}

In this work we observed and analyze some relevant biomedical literature involved in clinical pharmacist presence in some medical team and the results obtained from an practical experience in order to produce a global conclusion.

\section{Results}

\section{From Literature}

[1] in 2007clinical pharmacy service, pharmacy staffing, and hospital mortality rates. : " in 7 hospital, clinical pharmacy service reduces mortality rates." In a significant way [2] in "Pharmacist's effect as team members on patient care: A systematic review and meta-analyses": "Pharmacists provided direct patient care has favorable effects across various patient outcomes, health care settings, and disease states. (significant $p<0,005$ )" [3]. This paper wants to improve the pharmaceutical care application in countries with advanced healthcare system in order to provide a more rational drug therapy to patients. When this is not possible, it would be a good idea using the pharmaceutical care, in determinate populations such as: severe disease, critically ill, patients with multiple illnesses, transplants, immunosuppression, oncology or other serious conditions, at least when the treatments are very expensive." And "In these studies, we observe a general positive influence of pharmacist's presence in the medical equips also in different clinical outcomes" [3].

The relevance of pharmaceutical care to diagnostic imaging can be considered from 2 approaches. Diagnostic imaging modalities either are based upon or employ drugs. radio-pharmaceuticals are the key to nuclear medicine procedures and radiopaque contrast agents are essential in many radiographic studies. The principles and practice functions touted for therapeutic medications and therapeutic patient- management apply to drugs employed in diagnostic imaging, as well. Diagnostic imaging modalities are also intimately involved in determining the disease state in many patients. diagnostic imaging is utilized to follow the course of therapy; i.e., determining therapeutic outcomes. Pharmacists, not only specialists, must be knowledgeable of the role diagnostic imaging plays in pharmaceutical care and be prepared to provide the pharm. care in diagnostic imaging [4-5]. The intent of this work is to provide pharmacists with an introduction to the clinical pathology laboratory discipline. As clinical pharmacy services expand, interactions between pharmacists and the laboratory will increase. Laboratory results are an essential tool for pharmacists involved in monitoring the drug therapy and adjusting dosing regimens. Laboratory medicine is a complex and rapidly changing field with new analytical techniques - instruments and continually being developed. methodologies vary greatly from one lab. to another and even within the same laboratory from time to time. Quality control QC are necessary to ensure accurate and reliable results. The medical technologists who staff clinical laboratories are highly trained professionals. Pharmacists should utilize the medical technologist as a consultant on the interpretation / limitations of laboratory tests. there are many areas, such as therapeutic drug monitoring TDM , in which the pharmacist can serve as a consultant to the laboratory. Pharmacists involved in patient care will benefit from a greater understanding of the clinical laboratory and may also find new opportunities for the clinical pharmacy practice and interaction with other health care professionals [5].

According Clinical Pharmaceutical Care: A New Management Health Care Discipline in 2016 UKJPB "The development of clinical pharmacy in 1960-70s and pharm. care in 1990 has represented an interesting innovation in pharmacy field. clinical pharmacy and Pharm. care showed some practice critical limits:

(a) clinical pharmacy approach is more oriented to the clinical population needs or pathology but is not strictly request to manage at the level of every single clinical patient case.

(b) In hospital setting Pharmaceutical care can not to be applied to the entire patient for economic reason (limited number of pharmacist applied).

To give a more rationale priority of actions and to select the patient to be seen in priority way we think a good solution to apply the principle of pharmaceutical care with the instrument of clinical pharmacy into a new management system [6].

According article Infectious Disease Pharmaceutical Care: Analyzing the article reported in this work we can see a relevant role of clinical pharmacist in field of the infectious disease [7]. Infectious clinical pharmacist provides direct patient care in different inpatient settings (internal medicine a, critical care hemology/ oncology, solid organ transplant with other, as well as outpatient settings such as HIV clinics). Antibiotic stewardship/surveillance programs can be considered efficacy instruments available today as well as a guideline of protocols, procedure, EBM criteria and many others. Clinical pharmacokinetic consultant service, microbiologic and laboratory assay and assessment, scientific drug information, toxicity management, ADR, interactions, medicinal chemistry competences are the core curriculum of clinical pharmacist course and for this reason the permanent presence of clinical pharmacists results in general positive outcome in many clinical equip" $[7,8]$. To review the effects of pharmaceutical care on hospitalizations, mortality and clinical outcomes in patients. "Systematic searches were conducted in biomedical database like MEDLINE, EMBASE and International Pharmaceutical Abstracts (IPA) databases to identify studies that were published between 2004 and January 2017. Studies included in this review were randomized controlled trials (RCTs) that spanned across both community and hospital settings. Using strict inclusion/exclusion criteria studies were 
included if they reported level 1 or 2 outcomes in the hierarchy of outcome measure i.e. clinical and surrogate outcomes (e.g. blood pressure (BP) control, blood glucose level, cholesterol BMI).

54 RCTs were included in the present review. 46 of these studies ranked high- quality according to the Jadad scoring system. Studies were categorized into their general condition groups. Interventions in patients with diabetes, depression, respiratory disorders, cardiovascular disorders, epilepsy, osteoporosis, and interventions in older adults were identified. In the majority of studies pharmaceutical care was found to lead to significant improvements in clinical outcomes and/or hospitalizations when compared with the non-intervention group. Some conditions had a large number of RCTs, for example for cardiovascular conditions and in diabetes. Statistically significant improvements were seen in the majority of the studies included for both of these conditions, with studies indicating positive clinical outcomes and/ or hospitalizations rates. Within the cardiovascular CV condition, a subset of studies, focusing on cardiac heart failure and coronary heart disease, had more mixed results. In other conditions the number of RCTs conducted was small , the evidence did not show improvements after pharmaceutical care, Example in depression, osteoporosis, and epilepsy. The majority of interventions were face to face interactions with patients, whilst a smaller number were conducted via phone and one with a web-based system. Patient education was a key component of most interventions, verbal and/ or written. Longitudinal data, post intervention cessation, was not collected in the majority of cases.

RCTs conducted to evaluate pharm. care appear to be effective in improving patient short-term outcomes for a number of conditions including diabetes and CV conditions, other conditions such as depression are less well researched" [8,9]. "Pharmacists were consulted by the physicians to correct any drug-related issues that they suspected may cause or contribute to a fatal outcome in the cardiology ward. A total of 1,541 interventions were suggested by the clinical pharmacist in the study group; 1,416 (92.0\%) of them were accepted by the cardiology team, and violation of incompatibilities had the highest percentage of acceptance by the cardiology team. All-cause mortality was $1.5 \%$ during Phase I (pre-intervention) and was reduced to $0.9 \%$ during Phase II (post-intervention), and the difference was statistically significant $(\mathrm{P}=0.0005)$. After PS matching, all-cause mortality changed from $1.7 \%$ during Phase I down to $1.0 \%$ during Phase II, and the difference was also statistically significant $(\mathrm{P}=0.0074)$ " $[9,10]$. Clinical pharmacists did not perform any interventions during the phase 1 (pre-intervention) and consulted with physicians to address drug related problems (DRPs) during phase 2 (postintervention). The main outcome was a decrease in mortality from AMI. The 2 phases were compared using propensity score matching (PSM).

\section{Results Obtained}

1388 interventions were suggested by clinical pharmacists during phase 2, of which 1239 (89.2\%) were accepted. Logistic regression- analysis demonstrated that interventions of clinical pharmacists were significantly associated with a reduced mortality in patients with both ST segment elevation myocardial infarction (STEMI) (OR 0.449; 95\% CI 0.296 to 0.680) and non-ST segment elevation myocardial infarction (NSTEMI) (OR 0.268; 95\% CI 0.125 to 0.572 ). Using PSM analysis, mortality reduced from $6.8 \%$ to $4.3 \%$ in STEMI patients $(\mathrm{P}=0.0034)$ and from $3.2 \%$ to $0.7 \%$ in NSTEMI patients $(\mathrm{P}=0.0202)$ after interventions. DRPs that caused or contributed to possible mortality were detected by clinical pharmacists in patients with AMI. Correcting these DRPs after pharmacists' interventions could result in a significant decrease in mortality" $[10,11]$. "This research -study was conducted with 13 health-system pharmacists and 5 local health managers that were involved in the CPS implementation. The pharmacists who consented to participate were those who agreed to implement CPS in their respective workplaces. In order to get a comprehensive understanding of the facilitators that influenced the CPS implementation process, the health-system pharmacists were divided into 2 groups:

(i) 7 accredited pharmacists, who fulfilled all steps of the process and implemented CPS in their workplaces; and

(ii) 5 non-accredited pharmacists, who did not complete all steps of the process and did not fulfill implement CPS in their workplaces setting. The local health managers were those who held important positions in the local healthcare network (directors, supervisors, and coordinators) during the implementation of CPS.

Regarding the structure of the local healthcare network, the organization of services, health facilities, and local pharmaceutical services was reported to be a facilitator. Some units presented infrastructure as a facilitator, with adequate - comfortable physical space for pharm. care (private acclimatized room), computer equipment, and clinical devices (tensiometer, glucometer, et other). "Accredited pharmacists reported facilitators related to their attitudes, as willpower, commitment, proactivity to learn and perform clinical activities. The motivation generated by the positive results of CPS boosted the progress of these services. Managers reinforced the availability and intellectual/personal commitment of pharmacists to obtain knowledge that would help the implementation of CPS. "A great facilitator, in the first place, was my willpower to want to do, to learn, despite the difficulties; I think the proactivity of each one was fundamental for this" (Accredited Pharmacy D).

The clinical profile was cited as facilitator. We defined this profile as a set of intrinsic characteristics of a professional who 
is motivated a, had an affinity for clinical activities, previous knowledge, skills and attitudes. Some pharmacists reported having a previously created clinical profile to act directly with the patient, which was stimulated by the CPS implementation process. lifelong knowledge has helped pharmacists in clinical practice and patient management. "I want to be with the patient, not only as a pharmacist, but like a human who wants to make a difference in the patient' life, since the time they are diagnosed until the moment they know that it is possible to live with the disease and have a good quality of life" (Accredited Pharmacist G).

The pharmacists' work process, integrating collaborative practices with other members of the health-care team, in a different setting was pointed out as important facilitator. access to medical records, previous pharmacist-patient interactions. "I have full access to the patients' medical records, and physicians are accessible. With RT [reference technicians] we can talk about patients, like any healthcare team" (Non-accredited Pharmacist C)

\section{Facilitators Related to Implementation Process of CPS}

Participants reported that assistance provided by supporters during the implementation of CPS was an essential facilitator, both in theoretical and practical training, as mentoring activities during pharm. care. The local health- manager also recognized the expertise and contributions of supporters in CPS implementation. the systematization of the pharmacists' work process through documentation in medical records helped assimilate and incorporate clinical activities into the work routine of the pharmacists.

\section{Strategies to Implement CPS Related to the Local Health- care Network}

I do not think [local health- management] giving us a glucometer in December is a support. Support is to be together, to attend. (Nonaccredited Pharmacist C).

\section{Strategies Related to the Pharmacists}

Pharmacists proposed strategies they themselves should develop to facilitate the implementation process. Accredited pharmacists reported pro-activity and humanized posture during clinical activities and recruited patients through an active search. The non-accredited pharmacists suggested delegating some activities to the pharmacy staff and delegating the recruitment of patients to the health-care team. Many strategies were cited that aimed at strengthening relations between pharmacists, the healthcare team, and patients. Thus, it was proposed that the pharmacy staff should be able to collaborate in logistic activities by sensitizing the healthcare team and patients, in addition to disseminating implemented CPS through lectures, waiting area approach, home visits, and shared care. Non-accredited pharmacists pointed out the importance of strategies that would help reconcile the time between the logistic and clinical activities and how to delegate logistic activities so that new activities required for CPS implementation process were incorporated.
"I used shared care with nurses and physicians as a strategy, because in this way they could understand the process, the service, and what could be the result they saw my way of intervening in care and then I created a bond for the next consultation." (Accredited Pharmacist B).

\section{Strategies Related to the CPS Implementation Process}

Some accredited pharmacists suggested some strategies that could be adopted in future implementation processes in local healthcare networks. According to them, it would be interesting to adapt the CPS according to social demands where the service will be offered and select the pharmacists according to their clinical profiles this could ensure better medication adherence and reduce the dropout rate during the process. "There was a lack of selection on the professionals' profile there were many professionals who actually started doing this because they were involved, but they had no "clinical profile", no capacity, or no help, either they had no infrastructure or, due to any other difficulty, they gave up the project and this was discouraging for other professionals too" (Accredited Pharmacist C). This research study identified facilitators and strategies of the implementation of CPS. Most of the positive experiences were related to the clinical skills and pro-active attitudes of pharmacists. These findings may support pharmacists and health managers to implement CPS in health systems [11,12]. Critical care pharmacy services in the ICU have expanded from traditional dispensing responsibilities to being recognized as an essential component of multidisciplinary care for critically ill patients.

Augmented by technology and resource utilization, this shift in roles has allowed pharmacists to provide valuable services in the form of assisting physicians and clinicians with pharmacotherapy decision-making, reducing medication errors, and improving medication safety systems to optimize patient outcomes. Documented improvements in the management of infections, anticoagulation therapy, sedation, and analgesia for patients receiving mechanical ventilation and in emergency response help to justify the need for clinical pharmacy services for critically ill patients. Contributions to quality improvement initiatives, scholarly and research activities, and the education and training of interdisciplinary personnel are also valued services offered by clinical pharmacists. Partnering with physician and nursing champions can garner support from hospital administrators for the addition of clinical pharmacy critical care services. The addition of a pharmacist to an inter-professional critical care team should be encouraged as health-care systems focus on improving quality and efficiency of care delivered to improve the patient outcomes. In a single-centered research study, the direct contribution from the clinical pharmacist through interpretation of microbiology results, review of patient records, and recommendation of antimicrobial agents was associated with a reduced time to administration of the optimal antimicrobial therapy from 64.736 .8 to 39.315 .5 h $(\mathrm{P}<$ $.002)$. 
Additionally, de-escalation of antimicrobial therapy Over a 2-year period, the pharmacist reviewed 1,329 orders for restricted and nonformulary antibiotics for appropriateness on the basis of presumed infection, culture results, formulary availability, and dosing. The pharmacist recommended changes in . $50 \%$ of orders, with most changes related to spectrum of activity, formulary substitution, or dose adjustments. These interventions were associated with shorter lengths of stay (10.8 days vs 13.2 days, $\mathrm{P}<, .001)$ and decreased mortality $(6.61 \%$ vs $8.28 \%, \mathrm{P}=$ .007) compared with the period before implementation of an antimicrobial control program directed by a clinical pharmacist. The overall effect of a pharmacist on clinical outcomes in patients with known infections was investigated by combining data from the 2006 survey of ICU pharmacy services previously described with International Classification of Diseases, 9th Revision, Clinical Modification codes and data from the Centers for Medicare and Medicaid Services. Compared with ICUs with clinical pharmacists, mortality rates in ICUs that did not have clinical pharmacists were higher for nosocomial-acquired infections, community acquired infections, and sepsis by $23.6 \%$ (386 excess deaths, $\mathrm{P}, .001$ ), $16.2 \%$ (74 excess deaths, $\mathrm{P}=.008$ ), and $4.8 \%$ (211 excess deaths, $\mathrm{P}=$ .008), respectively. Similarly, ICU length of stay was longer for all infection categories by $7.9 \%$ (14,248 excess days, $\mathrm{P}<.001), 5.9 \%$ $(2,855$ excess days, $\mathrm{P}=.03)$, and $8.1 \%(19,215$ excess days, $\mathrm{P}<001)$, respectively.

A survey and database review of 242,704 Medicare surgical patients from 806 hospitals showed that pharmacist managed antimicrobial prophylaxis was associated with significant improvement in clinical outcomes, such as decreased surgical site infections and length of stay. In hospitals that did not offer pharmacist-managed antimicrobial prophylaxis, annual death rates were 52\% higher, with 105 excess deaths (OR, 1.54; 95\% CI, 1.46-1.63; $\mathrm{P}<, .0001$ ); length of hospital stay was $10.2 \%$ longer, with 167,941 excess patient days ( $\mathrm{P}<, .0001)$; and infection complications were $34.3 \%$ higher (OR, 1.52; 95\% CI, 1.40-1.66;

$$
\mathrm{P}<.0001) \text { than in those with pharmacist involvement [12]. }
$$

\section{According publication Sharing Economy and Healthcare Today}

The aim of this work was to analyze the relationship between professional social- media use and the healthcare in sharingeconomy time when used in healthcare field and specifically in pharmaceutical hospital settings [13]. The innovation introduced with bio-medical databases has improved research works with rapid steps in all kind of scientific researches areas tools as Internet, search engines and professional social media in sharingeconomy time have brought a great development in the way of rapidly connecting with the professionals. The researchers and professionals all over the world in last 2 decades has been great possibility to sharing their experience more than past with improving in their results. it is very interesting to see how they are playing a crucial role today healthcare through this rapid development. For example, LinkedIn, Slide share, ResearchGate, PubMed, YouTube, NCBI, Facebook, Skype et others social media . This has really brought a revolutionary change in the field. The possibility to bridge researchers and professionals with similar interests, discipline with more rapid development, never seen in last two decades. We think that using sharing economy instruments we can reduce healthcare costs about 30-40\%" [13,14]. "Pharmacists in Japan currently play a crucial role in patient hospital care. Their responsibilities include filling prescriptions, checking a patient's medication history, and providing appropriate information to other health care workers. More importantly, pharmacists' interventions can result in reductions in ADRs and, and in cost savings. This study aimed to determine the economic value of such interventions at a hospital in Japan.

At a single Japanese hospital, was analyzed 1452 pharmaceutical interventions by pharmacists, including recommending antibiotic dosage regimens, attending ward rounds with multidisciplinary health providers, providing drug information, and reporting ADRs. We classified the interventions into 13 categories. Using data from the Pre-AVOID Report by the Japanese Society of Hospital Pharmacists, along with previous studies, we estimated the cost savings of the interventions. Various savings could be realized through appropriate interventions by hospital pharmacists. Based on the amount paid by the Pharmaceuticals and Medical Devices Agency, we calculated the cost savings associated with preventing serious ADRs as 21,400 USD (\$) per case. The cost savings for recommendations related to transvenous antimicrobial therapy amounted to $\$ 1900$ per patient. Pharmacists' interventions were able to prevent 12 cases of serious ADRs. Determining the economic value of pharmacists' interventions is an important means of appraising the current role of the hospital pharmacists. Our evaluation demonstrates the positive economic effects of pharmacists' interventions in hospital setting "[14].

\section{According Pharm Care and Toxicology}

"Poisoning often is a rare event, but in some cases whit critical consequences and so the right diagnosis and therapy is a golden endpoint. The toxicology medical tram-equip must be multiprofessional. Observing the results of bibliography citied in this works and some university toxicology programs for pharmacists when observe that the clinical pharmacist presence in stabile way in toxicologist medical team give improving in clinical outcomes. Antidotes are used not often but rarely, and physicians need rapid information also in medicinal chemistry and toxicology field. The management of the systems must involve clinical and logistic

pharmacist. The pathology, toxicology, pharmacology , medicinal chemistry competence of clinical pharmacist added to the emergency and ICU physician's competences can be the right keywords. 
The skills requested to the clinical pharm. in order to works in efficiently way in toxicological medical team are pro-activity, learn about error, critical thinking, collaborative, approach, management ability, problem solving risk management (therapy errors management, some example and causes. illegible handwriting), we think that in order to have a more and efficacy inclusion of clinical pharm. in the toxicologist equip also psychological and behavior specific skill are useful instruments. New tools as professional social media can give more opportunity to meet researcher in healthcare field. Luisetto et al int. journal of economics and management sciences 2016, Instrument to rapid share the information between the healthcare professionals and to transfer research activities to practical settings" $[15,16]$. "Emergency Medicine clinical pharmacists services in the USA were first described in the biomedical literature in the 1970s. Initially, the role was confined mainly to medication distribution, but has evolved significantly into clinical practice.

The Inclusion of clinical pharmacists in EM has been supported by relevant org. as The American Society of Health-System Pharmacists, Agency for Healthcare Research and Quality (AHRQ), American College of Medical Toxicology and the Am. College of Emergency Physicians "The contribution of clinical pharmacists on multidisciplinary teams has been shown to reduce patient mortality, hospital readmissions 4 and medication errors. Perhaps the biggest critique against having EM clinical pharmacists is the global cost.

Pharmacists are an expensive resource and institutions may be concerned about whether the cost justifies the benefit. multiple studies have demonstrated significant cost avoidance with pharmacist presence in the ED, ranging between \$1-\$1.7 million saved per year well beyond what is needed to support a pharmacist's salary. These savings were associated with adherence to evidence-based EB and institutional guidelines, reduction in medication errors, timely institution of drug therapy, and use of cost-effective drugs and treatment"[16,17]. In article Integrating a clinical pharmacy service in the ED Related to the Critical medications : "Interventions correcting situations regarding a combination of failure to prescribe a critical drug or failure to administer a prescribed critical drug comprised a total of 215 (172 failure to prescribe, 43 critical drug not given). his comprised a total of $36.7 \%$ of the total interventions " $[17,18]$ and related to medication error "To evaluate the clinical relevance of pharmacist intervention on patient care in emergencies, to determine the severity of detected errors. to analyse the most frequent types of interventions and type of drugs involved and to evaluate the clinical pharmacist's activity. A 6-month observational prospective study of pharmacist intervention in the Emergency Department (ED) at a 400-bed hospital in Spain was performed to record interventions carried out by the clinical pharmacists. We determined whether the intervention occurred in the process of medication reconciliation or other activity, and whether the drug involved belonged to the
High-Alert Medications Institute for Safe Medication Practices (ISMP) list.

To evaluate the severity of the errors detected and clinical relevance of the pharmacist intervention, a modified assessment scale of Overage and Luke's was used. Relationship between clinical relevance of pharmacist intervention and the severity of medication errors was assessed using ORs and Spearman's correlation coefficient. During the observation period, pharmacists reviewed the pharmacotherapy history and medication orders of 2984 patients. A total of 991 interventions were recorded in 557 patients; $67.2 \%$ of the errors were detected during the medication reconciliation. Medication errors were considered severe in $57.2 \%$ of cases and $64.9 \%$ of pharmacist intervention were considered relevant. About $10.9 \%$ of the drugs involved are in the High-Alert Medications ISMP list. The severity of the medication error and the clinical significance of the pharmacist intervention were correlated (Spearman's $\rho=0.728 / p<0.001$ ). In this single centre study, the clinical pharmacists identified and intervened on a high number of severe medication errors. This suggests that emergency services will benefit from pharmacist-provided drug- therapy services [18]. In Rethinking the Hospital Pharmacist Service: Centralized Logistics- Ict Systems and Clinical Pharm.

"Analyzing the biomedical literature reported in this work and the results of a specific practical experience we can say that using drugs central logistics (hub and spoke pharmacy system) added to ICT instrument and clinical pharm. care and amore clinical pharmacy approach we can have reduced costs about $40 \%$. This result not only due by drugs and med. Devices costs rationalized but also by the reducing of med. errors, days of recovery and especially by improving clinical outcomes. the costs for an expensive transplant can be lost easy

if not applied efficacy pharmacological drug therapy (in example thinking about complex cardiac surgery or other critical and expensive patients situation).The clinical pharmacist competences/ skills added to ADVANCED ICT INTRUMENTS can improve the global clinical results.This is the real reason to shift from the classic logistic competences and skills of clinical pharmacist towards more and deep clinical tasks and to make an intensive ICT use as requested today" [19]. related an Emergency drug cabinet system practical experience : "We have also see in some practical experience that the introduce in daily works of some technologies make possible a right management (reducing total costs and with safety) in example in emergency drug cabinet systems EDCS "no near miss event or other patient risk or even fatal event was observed and was covered the the emergency need of drugs in the $99 \%$ of cases, and only 1 time was necessary the central pharmacy call." (The same results we have observed during the period of One year), we can have also a reduction in total costs providing an efficacy system .( about 50.000 euro in 5 years in an Italian public hospital 700 beds). 
Other ICT instruments are in examples dose unit system to provide single Unit of drugs (preventing waste or expiration of pharmacological therapies- full therapy traceability), informatics prescription software (to detect interactions, allergy, toxicity ),Informatics management of narcotic drugs or antidotes for right logistics and other" $[20,21]$. And in an practical hospital experience 2016: "Emergency drug hospital cabinet systems EDCS practical experience- PC (society of Italian hospital pharmacist POSTER ABSTRATC) MILAN national congress 2016 (this poster has win the best presentation award in the young pharmacist section) . In this practical example the ICT tool can make possible to have the real situation of the emergency drug (quali- quantitative) in every SPOKE and rapid information about emergency drugs position in order to achieve they in rapid way to have availability of other emergency drugs (h24 also with central pharmacy closed) in a safety way and containing costs. This experience is linked with a correct management of the systems with a really rational way and recognized officially by SIFO society of hospital pharmacist (Italy).

In this practical experience, no near miss event or other patient risk or even fatal event was observed related to emergency drugs stokes. During 6 month it was covered the emergency need of drugs in the $99 \%$ of cases, and only 1 time was necessary the central- pharmacy call by night or in week end. (The same results was observed during 1 year). Provincial public hospital with 700 beds and 4 hospital linked. This project was introduced by a multidisciplinary team according a risk management and ICT Approach. (Emergency and ICU clinicians, clinical pharmacist, informatics, engineers, nurse, toxicological med lab professionals and other). This systems in fact make possible to know rapidly the situation of expiration- time of all emergency drugs stoked and making easier the ordering process to have continuity in providing" [22,23]. The implementation of a comprehensive, 24-hour emergency department pharmacy program (EDPP) is described. "An EDPP was created at the Veterans Affairs San Diego Healthcare System to address deficiencies identified by the pharmacy service within the ED, including medication tracking, documentation of doses administered, formulary management. The documentation system used in the EDPP, a computerized spreadsheet and documentation cards, allowed the activities of the ED pharmacists to be tracked on a 24-hour basis. This type of data -collection allowed us to evaluate the impact of the program based on the quality of patient care delivered as well as cost. During the initial 6-month implementation period, the ED pharmacists recorded 9,568 interventions. The information from these interventions was used to assess the safety components and to estimate the cost avoidance of their activities. A staff satisfaction survey was also created to assess the pharmacist's impact on providers and nurses, as well as its effect on workflow within the ED. Among the many benefits realized, the EDPP improved the quality of patient care, decreased medication errors and patient wait times, improved the medication reconciliation process, enhanced formulary management, ensured prospective medication order review, and increased overall patient safety, as evidenced by the documented interventions and staff satisfaction survey. The projected cost savings for the medical center during the first year of EDPP implementation was calculated as $\$ 1,691,185$.

A tertiary care teaching hospital successfully implemented a 24-hour, comprehensive ED- pharmacy service that enhanced the efficiency - delivery of patient care and resulted in significant cost savings"[23,24]. "The Froedtert Acute Stroke Team (FAST) is composed of various health professionals who respond to stroke calls, but it does not formally include a pharmacist at this time. However, emergency department (ED) pharmacists have been actively involved in patient evaluation and facilitation of i.v. recombinant tissue plasminogen activator (rtPA) preparation and administration in the ED. ED pharmacists are qualified to dose and prepare rtPA, as well as screen for contraindications to the therapy. The primary objective was to compare the accuracy of rtPA dosing, mean door-to-rtPA time, and identification of contraindications to rtPA therapy when a pharmacist was present vs. absent in the ED. This is a retrospective study of 105 patients who received rtPA for acute ischemic stroke in the ED at a comprehensive stroke center from January 1, 2008 to October 1, 2012.: A total of 105 patients were included in this study. Dosing accuracy was similar when a pharmacist was present vs. absent (96.6\% vs. 95.6\%; $p=0.8953)$. The median door-to-rtPA time when a pharmacist was present was statistically significantly shorter than when a pharmacist was absent (69.5 vs. $89.5 \mathrm{~min}$; $\mathrm{p}=0.0027$ ). When a pharmacist was present, a door-to-rtPA time of $<60$ min was achieved $29.9 \%$ of the time, as compared with $15.8 \%$ in the pharmacist-absent group ( $p$ $=0.1087$ ).

Pharmacist involvement on stroke teams may have a beneficial effect on door-to-rtPA time and patient care in the ED" [24,25]. "Emergency department -based clinical pharmacy is a rapidly growing practice area that has gained support in a number of countries globally, particularly over the last 5-10 years. A systematic literature search covering the period 1995-2016 was conducted to characterize emerging EM pharmacist roles and the impact on patient outcomes. 6 databases were searched for research publications on pharmacist participation in patient care in a general ED or trauma center that documented interventions by ED-based pharmacists; 15 results satisfied the inclusion criteria. 6 reported studies evaluated EM pharmacist involvement in the care of critically ill patients, 5 studies evaluated antimicrobialstewardship program (AMS) activities via pharmacist review of positive cultures, 2 studies assessed pharmacist involvement in generating orders for nurse-administered home medications and 2 reviewed the publications focused on EM pharmacist involvement in management of healthcare-associated pneumonia and dosing of phenytoin. A diverse range of positive patient outcomes was identified. The included studies were assessed to be of low quality.

A systematic review of the literature revealed 3 key emerging areas of practice for the EM pharmacist that are associated 
with positive patient outcomes. These included involvement in management of critically ill patients, AMS roles, and ordering of home medications in the ED" $[25,26]$. "Emergency and critical care centers provide multidisciplinary therapy for critically ill patients by centralizing the expertise and technology of many medical professionals. Because the patients' conditions vary, different drug treatments are administered along with surgery. Therefore, the role of pharmacists is important. Critically ill patients who receive highlevel invasive treatment undergo physiological changes differing from their normal condition along with variable therapeutic effects and pharmacokinetics. Pharmacists are responsible for recommending the appropriate drug therapy using their knowledge of pharmacology and pharmacokinetics. pharmacists need to determine the general condition of patients by understanding vital signs, blood gas analysis results, etc. It is therefore necessary to conduct consultations with the physicians- nurses. The knowledge required for emergency medical treatment is not provided during systematic training in pharmaceutical education, meaning that pharmacists acquire it in the clinical setting through trial and error.

To disseminate the knowledge of emergency medical care to pharmacy students, emergency care training has been started in a few facilities. I believe that medical facilities and universities need to conduct joint educational sessions on emerge. medical care" [26,27]. "Recent evidence from conference proceedings at Guy's and St Thomas' Foundation Trust demonstrated that clinical pharmacists in the ED have been used for: allergy confirmation; providing a medicines safety barrier supporting safer prescribing in a high-risk environment; reduction in missed or delayed doses through early drug history identification; medicines reconciliation and supply of non-stock medicines; avoidance of adverse drug reactions; and provision of specific timely drug advice. This shows that role of the clinical pharmacist in the UK is and has been primarily concerned with pharm. care and medicines optimization, as opposed to truly integrating and supporting clinicians with managing clinical cases that are admitted into the ED.

Internationally, pharmacists also work in EDs, although they are known as 'hospital' or 'clinical' pharmacists, their roles are limited to pharm. care, review of medical care, or optimization of medication, and the role of the clinical pharm., mirroring that of a ward pharmacist who is present in the setting to screen physician orders and ensure medication safety. The American College of Emergency Physicians policy statement on clinical pharmacist services states that clinical- pharmacists serve a critical role in ensuring efficient, safe effective medication use in the ED and the College advocates health systems to support dedicated roles for pharmacists within the ED. The EM pharmacist should serve as a well-integrated member of the ED multidisciplinary team who actively participates in patient care decisions, including resuscitations, transitions of care, and medication reconciliation to optimize pharmacotherapy for ED patients. The policy statement also acknowledges variability in how this is delivered depending on the nature of the hospital setting "[27]. According article Steps and Impacts of Pharm. Care and Clinical Pharmacy Development on Clinical Outcomes 2016: A Historical Analysis Compared with Results, Clinicians Teamwork, 2016 : "During the periods of 1928 to 2016 a progressive and trendy development of clinical pharmacy and pharm. care was developed, as a new hospital discipline.

This approach was observed not only in the hospital settings, but also in community pharmacy in different ways, although with the same philosophy (patient-oriented pharmaceutical discipline) It was also observed a tendency of clinical pharmacist in field of medicine lab. and imaging for when they were related to the therapy and during its monitoring. Clinical pharmacists today are employed to reduce therapy errors and contain medication cost burdens. In addition to that what we have observed was a "general positive influence of the pharmacist's presence as a part of the medical team, on public health as well as in various clinical outcomes"; this according to clinical studies as systematic reviews, clinical trials and meta-analysis" [28,29]. "To evaluate the clinical pharmacists' interventions in an intensive care unit (ICU) setting with regard to their acceptance by the medical team, frequency, clinical significance, and targeted patient's outcomes.

This is a prospective, non-comparative, observational study evaluating clinical pharmacist interventions in an ICU setting from December 2002 to May 2003. The study was conducted in a 19-bed Cardiac-Surgery ICU at King Faisal Specialist Hospital and Research Center, a tertiary-care hospital in Riyadh, Saudi Arabia. The clinical pharmacist performed daily multi-disciplinary team rounds, with documentation of all his kind of interventions. On the same day, a physician, who is a part of the team, verified all interventions for validity and clinical significance. The institutional Office of Research Affairs approved the study. The clinical pharmacist intervened 394 times on the 600 patients [0.66 interventionper-patient]. The medical team accepted almost all interventions (94.3\%). The main drug-related problems were the following: no drug prescribed for medical condition (33.2\%), inappropriate dosing regimen (28.9\%), no indication for drug use (14.3\%). $55.7 \%$ of the interventions targeted enhancing therapeutic outcomes, whilst $21.8 \%$ of interventions resulted in the prevention of an adverse drug reaction.

The interventions that may have resulted in decreasing mortality, preventing, or reducing organ damage, or decreasing hospitalization, represented $8.1 \%$ of all interventions. Participation of a clinical pharmacist in the daily multidisciplinary team rounds in an ICU setting significantly reduces unfavorable morbidities and enhances therapeutic outcomes" [29,30]. "Pharmacist interventions and medication errors potentially differ between the People's Republic of China and other countries. This study aimed to report interventions administered by clinical pharmacists and analyze medication- errors in an intensive care unit (ICU) in a tertiary hospital in People's Republic of China. A prospective, 
non-comparative, 6-month observational study was conducted in a general ICU of a tertiary hospital in the People's Republic of China. Clinical pharmacists performed interventions to prevent or resolve medication errors during daily rounds and documented all of these interventions and medication errors. Such interventions and medication errors were categorized and analyzed. During the 6-month observation period, a total of 489 pharmacist interventions were reported. 407 (83.2\%) pharmacist interventions were accepted by ICU physicians. The incidence rate of medication errors was 124.7 per 1,000 patient-days. Improper drug frequency or dosing ( $\mathrm{n}=152,37.3 \%)$, drug omission $(\mathrm{n}=83,20.4 \%)$, potential or actual occurrence of adverse drug reaction $(n=54,13.3 \%)$ were the 3 most commonly committed medication -errors. Approximately $339(83.4 \%)$ medication errors did not pose any risks to the patients. Antimicrobials $(\mathrm{n}=171,35.0 \%)$ were the most frequent type of medication associated with errors. Medication errors during prescription frequently occurred in an ICU of a tertiary hospital in the People's Republic of China. Pharmacist interventions were also efficient in preventing medication errors" [30]. To review the effects of pharmaceutical care on hospitalizations, mortality and clinical outcomes in patients.

"Systematic searches were conducted in MEDLINE, EMBASE and International Pharmaceutical Abstracts biomedical databases to identify studies that were published between 2004 and January 2017. Studies included in this review were randomized controlled trials (RCTs) that spanned across both community and hospital settings. Using strict inclusion/exclusion criteria studies were included if they reported level 1 or 2 outcomes in the hierarchy of outcome measure i.e. clinical and surrogate outcomes (e.g blood pressure (BP) control, blood glucose level, cholesterol BMI). Each study was assessed for the quality using the Jadad scoring system. 54 RCTs were included in the present review. 46 of these studies ranked high quality according to the Jadad scoring system. Studies were categorized into their general condition groups. Interventions in patients with diabetes, depression, respiratory disorders, cardiovascular disorders, epilepsy, osteoporosis, and interventions in older adults were identified. In majority of studies pharmaceutical care was found to lead to significant improvements in clinical outcomes and/or hospitalizations when compared to the non-intervention group. Some conditions had a large number of RCTs, for example for cardiovascular conditions and in diabetes. Statistically significant improvements were seen in the majority of the studies included for both of these conditions, with studies indicating positive clinical outcomes and/or hospitalizations rates. Within the cardiovascular condition, a subset of studies, focusing on cardiac heart failure and coronary heart- disease, had more mixed results. In other conditions the number of RCTs conducted was small and the evidence did not show improvements after pharmaceutical care, i.e. in depression, osteoporosis, and epilepsy.

The majority of interventions were face to face interactions with patients, whilst a smaller number were conducted via the telephone and one via a web-based system. Patient education was a key component of most the interventions, either verbal and/or written. Longitudinal data, post intervention cessation, was not collected in the majority of cases. RCTs conducted to evaluate pharmaceutical care appear to be effective in improving patient short-term outcomes for a number of conditions including diabetes and cardiovascular conditions, however, other conditions as depression are less well researched. Future research should attempt to evaluate the conditions where there is a lack of data, whether the positive effects of pharmaceutical care persist in patient populations after the interventions cease and what the long-term clinical outcomes would be of continued pharm Care [31]. With increasing complexity of critical care medicine comes an increasing need for multidisciplinary involvement in care. In many institutions, pharmacists are an integral part of this team, but long-term data on the interventions performed by pharmacists and their effects on patient care and outcomes are limited. We aimed to describe the role of pediatric clinical pharmacists in pediatric intensive care unit (PICU) practice. We retrospectively reviewed the records of pharmacy interventions in the PICU at the Mayo Clinic in Rochester, Minnesota, from 2003-2013, with a distinct period of increased pharmacist presence in the PICU from 2008 onward. We compared demographic and outcome data on patients who did and who did not have pharmacy interventions during 2 periods (2003-2007 and 2008-2013) We identified 27,773 interventions by pharmacists during the 11-year period, of which $79.8 \%$ were accepted by the clinical equip. These interventions were made on 10,963 unique PICU admissions and prevented 5867 order entry errors. Pharmacists' interventions increased year over year, including a significant change in 2008. Patients who required pharmacy involvement were younger, sicker, and had longer intensive care unit, hospital, and ventilator duration. Average central line infections and central line entry rates decreased significantly over the study period. Increased pharmacist presence in the PICU is associated with increased interventions and prevention of adverse drug events. Pharmacist participation during rounds and order entry substantially improved the care of critically sick children and this should be encouraged" [32].

\section{A Practical Experience Results}

Location Pc AREA Italy Time of observation: from 2008 to 2018 Position observed: hosp. pharmacist antidothes responsible. (Provincial centre). Ward involved: EMERGENCY DEP., ICU ( related need also of cardiac and pediatric ICU) Results : No near miss event or other patient risk or even fatal event was observed Related use of antidotes or other emergencies drugs ( logistic or other related need ) . NO writed notify from other hospital office related any issue Related the antidotes management . Results was observed in this literature - studies a general positive influence of pharmacist 's presence in medical team also in different clinical outcomes. Related the results of a practical experience is possible to submit the efficacy of clinical pharmacist presence in toxicological medical 
team. Evidence of benefit is showed in biomedical literature and also in a practical experience reported. Evidence that can be shared also in other setting and other kind of hospital (moratlity rate, hospital readmission, medical errors)

The Clinical Pharmacist Interest is mainly on Some Drugs Classes Management in EM or ICU:

Like
a) Antidotes (A and B priority)
b) Hemo-derivates
c) Some parenteral antimicrobials
d) Anahestetics, Miorelaxants, analgesics and narcotics
e) Cardioactive
f) Electrolites
g) Antipertensive, diuretics
h) Antiepileptics

And Other necessary Emergency drugs to cover the various emergency need (compoundings, magistral formula) and few other classes.

\section{Discussions}

In emergency field and in ICU settings ( cardiac, pediatric or other ) we notice that clinical pharmacist can improve clinical and economic outcomes using the medicine laboratory and imaging knowledge for the relationship of this discipline in therapy and it's monitoring [4,5]. (In example microbiological data on antimicrobial resistance or sensibility and many other) From ASHP guideline :"EMERGENCY PHARMACIST provide many vital services within the Emergency dep . The central role of the EMP is to improve patient outcomes by improving patient safety, preventing medication errors, and providing optimized pharmacotherapy regimens and therapeutic outcomes through participation in direct patient care activities and quality-improvement initiatives in the ED. In addition, EMPs can provide education to members of the pharmacy department and other health care providers, as well as patients and their caregivers, and EMPs may participate in research and scholarly activities in the ED" According editorial the clinical pharmacist main focus is reprted : "We think that the main focus of the clinical pharmacist must be applied in priority way to the most critical patients in order to achieve the best results available . In this condition even benefit of 1 life achieved in mortality rate is a real golden endpoint (we can think for example to a paediatric poisoning, or severe infectious disease in pregnancy or the effect of inefficacy immunosuppressive therapy in transplanted et other) [10]. This can be considered in example as a reduction in NNT to improve a therapeutic strategy" [33].

\section{Conclusion}

Related the bibliography observed in this work we request a more involvement of clinical pharmacist in field as Emergency medicine, cardiac ICU, toxicology, infectious disease ,medicine laboratory and in imaging for clinical pharmaceutical care purpose. If in emergency medicine time is universally considered "TIME IS BRAIN" in stroke management (and the golden hour) the pharmacist clinical competencies is a fundamental fact for patient safety in the medical team (in example for administration of antibiotics in sepsis, thrombolythic therapy, antidotes administration and other) . The clinical pharmacist competencies related intensive care drugs, pharmacology, toxicology, medicinal chemistry, medical devices, infectious disease, cardiovascular, renal, epatic, neurology Bone marrow, pulmonary functions and many other in example TDM therapeutic drug monitoring Medicine lab competencies, Pharmacoeconomy are currently present in curricula studio rum of many clinical pharmacy International course. So Is clear that the clinical pharmacist and pharmaceutical care skills can be the right instrument to reduce mortality rate and cost containment. The same the economic results of this approach is a clear undeniable fact.

\section{References}

1. Bond C A , Raehl CL (2007) Pharmacy staffing, and hospital mortality rates: In seven hospital, clinical pharmacy service reduces mortality rates. In a significant way. clinical pharmacy service 27(4): 481-93.

2. Chisholm (2010) Pharmacist's effect as team members on patient care: Systematic review and meta-analyses. pharmacists provided direct patient care has favorable effects across various patient outcomes, health care settings, and disease states 48(10): 923-33.

3. M luisetto (2015) Pharmacist cognitive service and pharmaceutical care today and tomorrow outlook UKSPB 3(6): 67-72.

4. Stanley M Shaw (1994) Diagnostic imaging and Pharmaceutical Care. American J of pharmaceutical education 58: 2.

5. Howard P (1984) An introduction to the clinical laboratory for pharmacists. Howard P Hosp Pharm 19(6): 425-426

6. Luisetto M (2016) Ram Sahu Clinical Pharmaceutical Care: A New Management Health Care Discipline in 2016 UKJPB.

7. Luisetto M (2017) Infectious Disease Pharmaceutical Care: The Role of the Clinical Pharmacists to Improve Clinical Outcomes 2017. J Antimicrob Agents 3: 143.

8. Babar ZU, Kousar R, Murtaza G, Azhar S, Khan SA, et al. (2018) Randomized controlled trials covering pharmaceutical care and medicines management: A systematic review of literature. Res Social Adm Pharm 14(6): 521-539.

9. Xiao-bo Zhai, Zhi-chun Gu, Xiao-yan Liu (2016) Effectiveness of the clinical pharmacist in reducing mortality in hospitalized cardiac patients: apropensity score-matched analysis. Ther Clin Risk Manag 12: 241-250.

10. Xiao-Bo Zhai, Zhi-Chun Gu, Xiao-Yan Liu (2017) Original article Clinical pharmacist intervention reduces mortality in patients with acute myocardial infarction: a propensity score matched analysis. Eurp j of hosp pharmacy. 
11. Sheila Feitosa Ramos, Genival Araujo dos Santos, André Mascarenhas Pereira, Aline Santana Dosea, Kérilin Stancine Santos Rocha, et al. (2018) Facilitators and strategies to implement clinical pharmacy services in a metropolis in Northeast Brazil. BMC Health Serv Res 18(1): 632.

12. R Preslaski, Ishaq Lat, Robert MacLaren, Jason Poston (2013) Pharmacist Contributions as Members of the Multidisciplinary ICU Team Candice 144(5): 1687-1695.

13. Luisetto M (2016) Sharing Economy and Healthcare Today: ICT, Knowledge, Skills, Projects, Practical Experience in Improving Clinical and Economic Outcomes. J Bus Fin Aff 5: 207.

14. Daiki Yasunaga, Yuichi Tasaka, Satoshi Murakami, Akihiro Tanaka ,Mamoru Tanaka, et al. (2017) Economic contributions of pharmaceutica interventions by pharmacists: a retrospective report in Japan. J Pharm Policy Pract 10: 2.

15. Luisetto M (2016) Pharmaceutical Care and Toxicology, a Synergy in High Risk Situation. J App Pharm 8: 231.

16. Coralic Z, Hayes BD. Emerg Med J Month (2017).

17. (2016) Integrating a clinical pharmacy service in the ED16 PM Kunal Gohil MPharm PGDip Specialist ED Pharmacist, Claire Patel BPharm PGDip Divisional Lead Pharmacist for Medicines, Mojgan Sani DPharm MBA, Chief Pharmacist \& Clinical Director Medicines Optimisation, Nottingham University Hospitals NHS Trust; Visiting Professor University of Nottingham, and DMU, UK 05:28

18. Pérez-Moreno MA, Rodríguez-Camacho JM,Calderón-Hernanz B (2016) Clinical relevance of pharmacist intervention in an emergency department. Emerg Med J 34(8): 495-501.

19. Luisetto M (2017) Rethinking the Hospital Pharmacist Service Centralized Logistics- Ict Systems and Clinical Pharmaceutical Care Strategies as a Management Opportunity for Public and Private Institutions. JOJ Pub Health 2(3): 555589.

20. Luisetto M (2016) The emergency drug hospital system : l'armadio delle urgenze gestito dal Farmacista Ospedaliero Atti del XXXVII Congresso Nazionale SIFO Milano, 14 dicembre Confalonieri C . poster abstract sifo congress.

21. Luisetto M, Nili Ahmadabad (2016) Review article: Emergency Hospital Drug Cabinet Systems Managed by Hospital. J App Pharm 8: 4

22. Luisetto M, Mashori GR (2017) Intensive Care Units (ICU): The clinical pharmacist role to improve clinical outcomes and reduce mortality rateAn undeniable function. J Clin Intensive Care Med 2: 049-056.
23. (2009) Implementing a comprehensive, 24-hour emergency department pharmacy program. Am J Health Syst Pharm 66(21): 1943-1947.

24. Gosser RA, Arndt RF, Schaafsma K, Dang CH (2016) Pharmacist Impact on Ischemic Stroke Care in the Emergency Department. J Emerg Med 50(1): 187-193.

25. Roman C, Edwards G, Dooley M, Mitra B (2018) Roles of the emergency medicine pharmacist: A systematic review. Am J Health Syst Pharm 75(11): 796-806

26. Imai T, Yoshida Y, Yakugaku Zasshi (2016) Involvement of Pharmacists in Medical Care in Emergency and Critical Care Centers. 136(7): 967-972.

27. Matthew Aiello, David Terry, Nisha Selopal, Chi Huynh, Elizabeth Hughes, et al. (2017) The pharmaceutical journal Examining the emerging roles for pharmacists as part of the urgent, acute and emergency care workforce Clinical Pharmacist.

28. M Luisetto, B Nili-Ahmadabadi, L Cabianca, M Ibne Mokbul, (2016) Steps and Impacts of Pharmaceutical Care and Clinical PharmacyDevelopment on Clinical Outcomes 2016. A Historical Analysis Compared with Results, Clinicians Teamwork 1: 4-8.

29. Al-Jazairi AS, Al-Agil AA, Asiri YA, Al-Kholi TA, Akhras NS, et al. (2008) The impact of clinical pharmacist in a cardiac-surgery intensive care unit. Saudi Med J 29(2): 277-281.

30. Sai-Ping Jiang, Jian Chen, Xing-Guo Zhang, Xiao-Yang Lu, Qing-Wei Zhao, et al. (2014) Implementation of pharmacists' interventions and assessment of medication errors in an intensive care unit of a Chinese tertiary hospital. Ther Clin Risk Manag 10: 861-866.

31. Babar ZU, Kousar R, Murtaza G, Azhar S, Khan SA, et al. (2018) Randomized controlled trials covering pharmaceutical care and medicines management: A systematic review of literature. Social Adm Pharm 14(6): 521-539.

32. Sandeep Tripathi, Heidi M Crabtree, Karen R Fryer, Kevin K Graner, Grace M Arteaga, et al. (2015) Impact of Clinical Pharmacist on the Pediatric Intensive Care Practice: An 11-Year Tertiary Center Experience. J Pediatr Pharmacol Ther 20(4): 290-298.

33. Luisetto M, Nili-Ahmadabadi B, Mashori GR (2017) The Clinical Pharmacists Main Focus. J Appl Pharm 9: 114. (C)

To Submit Your Article Click Here: Submit Article

DOI: $10.32474 /$ LOJPCR.2019.01.000110

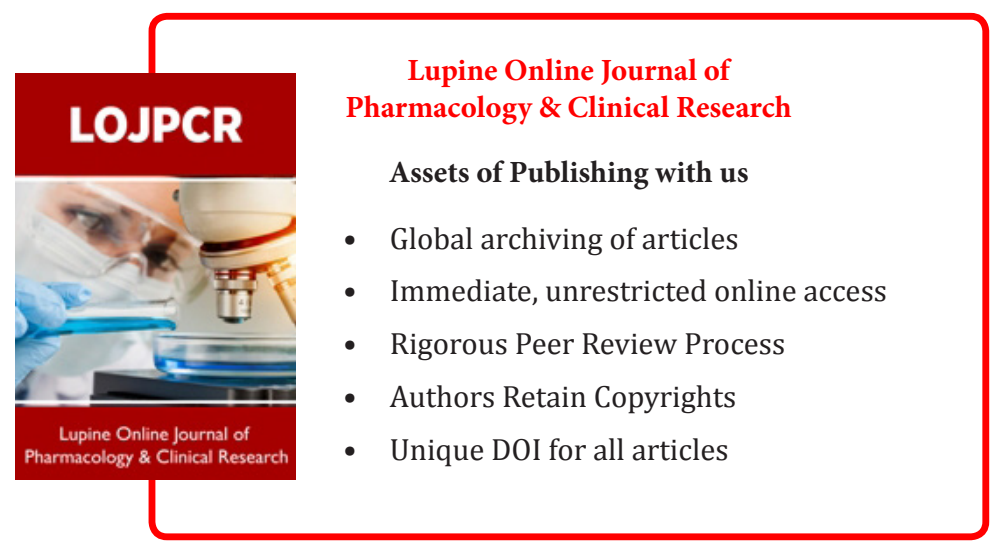

